\title{
NON-STATIONARY FLOW AROUND BUILDINGS DURING RUN-UP OF TSUNAMI WAVES ON A PLAIN BEACH
}

\author{
Nils Goseberg ${ }^{1,2}$ and Torsten Schlurmann
}

\begin{abstract}
This paper presents an experimental investigation that focuses on some predominant flow features that arise around surface mounted vertical obstacles which are exposed to a transient flow. The flow under investigation is caused by a tsunami-like long wave that climbs up a 1:40 sloping plain beach. In this study the wave height in offshore waters is varied. A single obstacle of $10 \mathrm{~cm}$ width as well as side-by-side arrangement of two identical square obstacles with different spacing are considered at an approximate length scale of 1 in 100. The analysis reveals important flow features around the various obstacle configurations. Particular emphasize is laid on the spatiotemporal evolution of the wake angle that linearly increases over time irrespective of the obstacle spacing. The growth rate of the wake angle reciprocally depends on the gap ratio over the investigated range of $g^{*}=0.0$ to $g^{*}=3.0$.
\end{abstract}

Keywords: tsunami; long wave; wave run-up; wake angle; obstacle; wave-structure interaction

\section{INTRODUCTION AND MOTIVATION Introduction}

In light of the disastrous consequence by major tsunami such as the 2011 Tohoku-Oki tsunami, the understanding of the generation, evolution and run-up of long waves has grown rapidly. On the one hand, the quantification of the maximum run-up of long waves on natural beaches and the determination of related hydrodynamic forces on the other hand are one of the quintessential questions. In this context it is of interest how urbanized coastal areas with macro-roughness (MR) elements (Cox et al., 2009) interact and potentially reduce those hazardous water levels and flow velocities. The severe inundation of the Sendai coast documented by a post-tsunami survey team suitably exemplifies this type of wave-structure interaction where houses and multi-storey buildings withstand the tsunami-induced current until they either collapsed or were washed away ( $\mathrm{Li}$ et al., 2011). Beside those houses that were damaged there is also evidence that many reinforced houses were capable to withstand the strong wave attack. In this regard, it is of interest how the flow field around beachfront development temporally develops during the run-up of long waves for further studies, e.g. to assess scour phenomena. Secondly, it is currently unclear how wake effects behind vertical rectangular structures and subsequent sheltering further influence water levels and forces in its flow path. To investigate such wave-structure interaction, hydraulic model tests depict a suitable method to assess the propagation and run-up of long waves climbing up a sloping beach as well as the flow around solid objects onshore.

\section{Related literature}

The flow around surface-mounted bluff bodies is subject to numerous studies across various disciplines (e.g., hydrodynamics (Sumer et al., 1997), aerodynamics (Ayukawa et al., 1993), geophysics (Cui and Gray, 2013)). So far, circular bluff bodies received by far more attention in literature compared with rectangular cylinders due to the applicability to many practical problems in mechanical, ocean or hydraulic engineering. However, flow around square cylinders is also of interest in many architectural and engineering technology applications; examples are the flow around cofferdams or non-optimized rectangular bridge piles placed in rivers, the study of flood hazard in urban settings, the inundation of coastal stretches from tsunami waves (Taubenböck, Goseberg, Setiadi, et al., 2009; Taubenböck, Goseberg, Lämmel, et al., 2013) or the interaction of wind fields with man-made development (Wong et al., 1995).

A free-surface flow interacting with a single bottom-mounted vertical object results in some typical flow features which may render secondary effects such as vortex shedding in the lee-wake, scour evolution at flume bottom or structural response in the form of oscillation induced from vortex shedding frequency to the adjacent flow field around the object of interest. The presence of most flow features is governed by the Reynolds $(R e)$, Froude $(F r)$, Keulegan-Carpenter $(K C)$ or Strouhal $(S t)$ numbers. In the upstream vicinity of the bluff body, a stationary approach flow results in a bow-like wave in the form of a shock commencing in the horizontal at the front face of a square cylinder and being advected by the velocity field and the cross-flow pressure gradients to both sides of the obstacle (Mignot and Riviere, 2010). In this case the bow wave is also stationary. Depending on the Froude number, shape and location of the bow wave changes. In the upstream near-bed region flow separation is reported which leads to

\footnotetext{
${ }^{1}$ Franzius-Institute for Hydraulic, Estuarine and Coastal Eng., Leibniz University, Nienburger Str. 4, Hanover, 30167, Germany

2 Department of Civil Engineering, University of Ottawa, 161, Louis Pasteur St., Ottawa, Ontario, K1N 6N5, Canada

${ }^{3}$ Franzius-Institute for Hydraulic, Estuarine and Coastal Eng., Leibniz University, Nienburger Str. 4, Hanover, 30167, Germany
} 
a horseshoe vortex system (Dargahi, 1989; Mignot and Riviere, 2010) not only for stationary approach flows but also for oscillatory flow and wave-current flows (Sumer et al., 1997) whose occurrence then greatly depends on the $\mathrm{KC}$ number regime. As another important flow feature in the form of a hydraulic jump is reported by Mignot and Riviere (2010) in supercritical flows due to the decelerating effect of the obstacle front face. At some distance upstream deceleration grows up to the point where subcritical conditions are reached; this is then reflected by a two-dimensional (2D) hydraulic jump. Mignot and Riviere (2010) summarized that the detachment length of the upstream toe of the observed hydraulic jumps and the separation point of the near-bed horseshoe vortex changes with respect to either laminar or turbulent inflow conditions during their experiments.

Aerodynamic studies concerning air flow around square cylinders bring about the vortical structure of the flow field under turbulent conditions. Sousa (2002) schematized the potential vortical structures when a square object interacts with a wind field by means of reconstructing the flow field from 2D digital partical image velocimetry into horseshoe, wake, roof and side vortex. Moreover, interferences that occur between square cylinders arranged in a group can be studied with air flow while the centre-to-centre distance is varied (Alam et al., 2011). The authors classified four distinct flow regime in the wake region that largely correspond to the relative gap width; in particular, form drag tends to decrease for a relative gap ratio of 1.2 to 3.0. Similar conclusions with respect to mode classification, time-averaged drag coefficients and vortex shedding frequencies were drawn by Yen and Liu (2011). In contrary to the surrounding velocity fields applied in aerodynamics that totally envelop the obstacles, hydrodynamic studies offer set out to simulate flow around (and not over) such structures. This difference is correlated with changes in the velocity fields and its turbulent structures around the obstacles; some analogies from aerodynamics might hold although differences are very likely when the obstacle is arranged in a fluid surface-piercing manner.

To the authors' knowledge only few studies focus on the interaction of a free-surface water flow with single or grouped square cylinders. Malavasi and Guadagnini (2007) investigate some features appearing during interaction of a horizontal square cylinder with an open-channel flow, e.g. mimicking a bridge deck, that is bounded by the free surface and the bottom in the vertical direction. Significant differences are found in this configuration in terms of form drag, lift coefficients and vortex shedding frequency or $S t$ number respectively compared with unbounded flows. Distortion of the free surface has a significant effect on the flow field in the vicinity of the obstacle and a dependance of the force coefficients and the vortex shedding frequency on the $R e$ is revealed. In addition, Singha and Balachandar (2011) confirm the quantitative differences between bounded and unbounded flows with respect to the velocity deficit which appears during the interaction of an open channel approach flow with a sharp-edged bluff body. Although the flow considered is already in a shallow regime, it is not apparent that flow transition in the wake region is found and thus, for this case only weak variations of the free surface are generation. Also, neither the approach flow velocity nor the water depth was varied over time as is the case for some relevant transient flow phenomena such as the wave run-up and dynamic overflow of coastal stretches as a consequence of a tsunami or dam-break event. More similar to natural hazardous flows as induced by earthquake-induced tsunami or dam-break is the work of Pagliara et al. (2011) who reports on supercritical flow behind chute piers resulting in spatially varying flow pattern commonly termed 'rooster tail'. The associated process of flood release of a dam is usually only weakly time-dependent and the flow along the chute is mostly driven by gravity. However, this flow pattern appears to be similar to those observable during the interaction of an approaching, shoreward propagating tsunami wave that eventually interacts and flows around coastal development as reported by Goseberg and Schlurmann (2012); Goseberg (2013).

\section{Objective}

The objective of this paper is thus to investigate experimentally how flow pattern around one or more bluff body -here a square cylinder of finite height- evolve for a transient free surface approach flow. The flow is abstracted with the particular aim to mimic hazardous currents as induced by tsunami or dam-break and aims at gaining further inside into the flow-structure interaction. It is meant to facilitate estimation of appropriate form drag values, e.g. for comparison with numerical models; so far, those flows differ from most of the flow conditions reported in literature with respect to temporal changes of water depth and flow velocity. For the particular case of tsunami hazard, flow reversal can be observed as well which in addition is driven by gravitational acceleration during the run-down phase of the long wave motion. Besides the qualitative description of the flow pattern, emphasis is laid on the description of the evolution of the wake angle that changes with respect to time and with the decreasing run-up velocity. 


\section{EXPERIMENTAL SETUP}

\section{Wave flume and wave generation}

The experiments reported herein were carried out in the closed-circuit wave flume at the Franzius-Institute for Hydraulic, Estuarine and Coastal Engineering, Leibniz University Hanover. A detailed description of the wave flume, its arrangement for the experiment and the wave generation by means of high-capacity pipe pumps adjusted by a PID controller is given in Goseberg (2012); Goseberg, Wurpts, et al. (2013). At one side of the closed-circuit flume a plain beach section with a slope of 1 in 40 is constructed. The surface of the beach consists of smooth alloy board which is sealed to the flume walls by silicone. Leading depression long waves of sinusoidal shape were generated for the tests. Table 1 summarizes the waves parameters of the applied waves. Amplitude of the two different sinusoidal waves was varied while period was kept constant. The real-time feedback mechanism of wave generation which compares set point and actual values of water level at the pump outlet was adjusted in calibration runs until a satisfactory level of agreement was achieved. Standard deviation for the two offshore waves according to Table 1 are $\sigma=0.0052 \mathrm{~m}$ for experiments no. $1-4$ and $\sigma=0.0065 \mathrm{~m}$ for experiments no. 5-8. While largest deviations between actual and set point values of surface elevation were measured for wave crest, absolute values are considered small enough for the objective of the study. However, some of the experimental runs were slightly biased by some short frequency, small amplitude 'riding' waves which were generated especially at the time of maximum wave crest. Influence and significance of those oscillations will be discussed below. Positions in this paper are indicated by dimensionless $\mathrm{x}$ and y coordinates according to the following nondimensionalization on the basis of the still water depth $h=0.3 \mathrm{~m}$ :

- shoreward positive direction $\hat{x}=\frac{x}{h}$, still water line is at $\hat{x}=39.80$, zero at toe of the slope

- lateral positive direction $\hat{y}=\frac{y}{h}$ with zero at symmetry axis of the slope

\section{Instrumentation}

Instrumentation of the experiments is equal to the instrument setting depicted in Goseberg (2013) and basically consists of pressure sensors, wave gauges and an electromagnetic probe. For the analysis of the velocity field around the macro-roughness elements and the wake formation behind the obstacles an overhead CCD camera (Basler AG) was applied which captured image frames at a frame rate of $32 \mathrm{~Hz}$. Subsequently, compressed, synchronized images were used for Particle Image Velocimetry (PIV) technique (Sveen, 2004) and to manually deduce the wake angle. Sesame seeds were utilized as tracers throughout this study which were placed on top of the water surface and during the experimental runs manually. In advance of the PIV analysis, images were barrel-corrected and macro-roughness elements were masked. PIV was conducted with a sub-window size of $[80,80 ; 80,80 ; 40,40 ; 40,40]$ pixels, an overlap of 0.75 between the images and subsequent filtering of the resulting velocity fields.

\section{Obstacle arrangements and general settings}

Four different spacing configurations of the obstacles were tested during wave attack. Details are shown in Figure 1. Spacing distance can be dimensionslessly expressed by a gap ratio $g^{*}$ which is defined as follows:

$$
g^{*}=\frac{g}{w}
$$

where $g$ denotes the spacing between the square cylinder surfaces and w is the diameter which is kept constant throughout the experiments at $0.1 \mathrm{~m}$. Obstacles were placed at a distance of $0.3 \mathrm{~m}$ from the shoreline in the landward direction. According to Tab. 1, gap ratios $g^{*}$ varying from 0.0 to 3.0 were this tested.

As the experiments were accomplished in a closed-circuit flume with cross-sectional width of $1.0 \mathrm{~m}$, gap ratios larger than 3.0 were not possible without biasing influence of boundary effects on the lee-side wakes. It remains therefore unsolved at which gap ratio side-by-side effects between the investigated cease to play a dominant role to the flow field and wake angle evolution.

\section{FLOW DESCRIPTION}

As a transient flow induced by long waves running up a plain beach approaches a surface mounted rectangular object which emerges above the free surface, a complex, a characteristic transient flow 


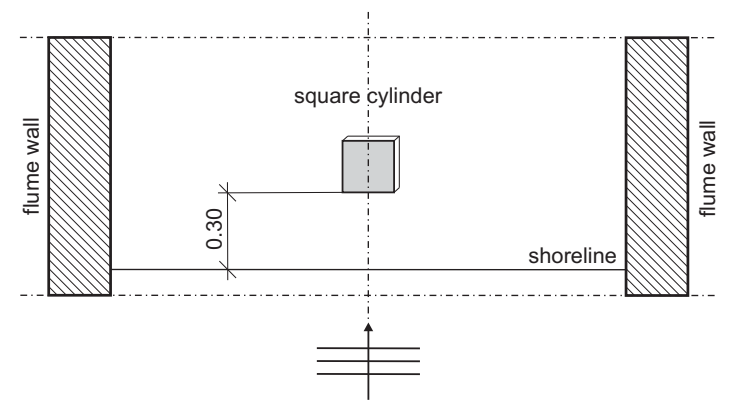

(a) $g^{*}=0.00$

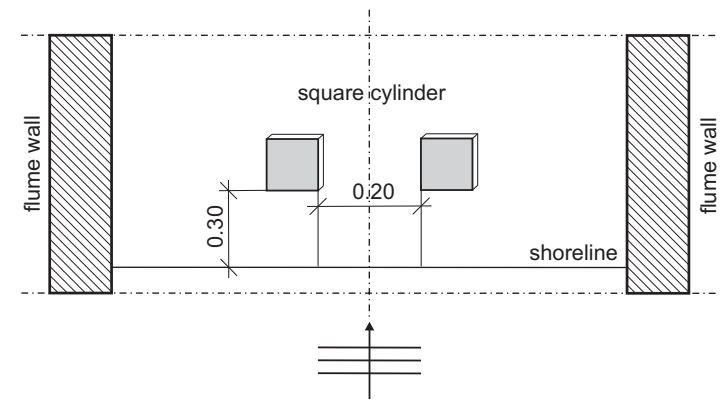

(c) $g^{*}=2.00$

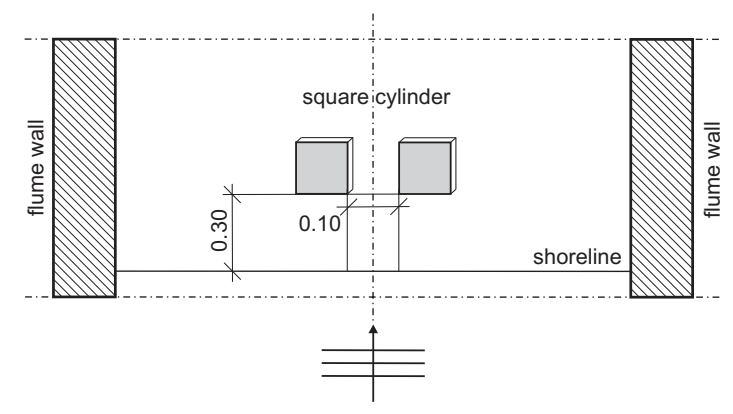

(b) $g^{*}=1.00$

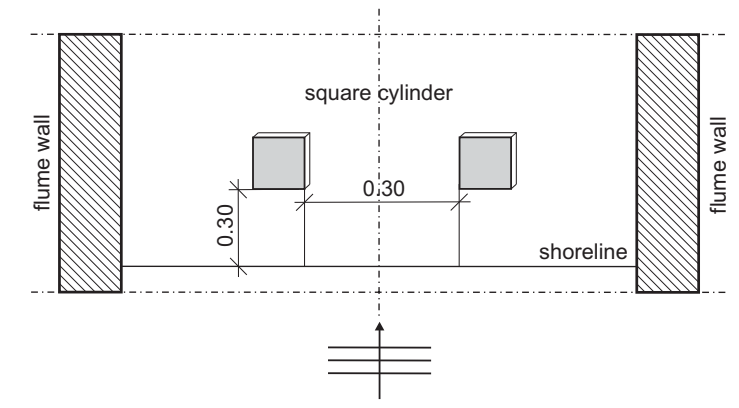

(d) $g^{*}=3.00$

Figure 1: Tested gap spacings $g^{*}=0.00-3.00$

Table 1: List of experiments with parameters of the applied waves at a deep water depth $d=0.3 \mathrm{~m}$ and the gap ratios chosen

\begin{tabular}{|c|c|c|c|c|c|c|c|c|c|}
\hline $\begin{array}{l}\text { No. } \\
{[]}\end{array}$ & $\begin{array}{l}\text { gap spacing } \\
{[\mathrm{m}]}\end{array}$ & $\begin{array}{l}\text { gap ratio } \\
{[]}\end{array}$ & $\begin{array}{l}\text { amplitude } \\
\text { [m] }\end{array}$ & $\begin{array}{l}\text { period } \\
{[\mathrm{s}]}\end{array}$ & $\begin{array}{l}\text { celerity } \\
{[\mathrm{m} / \mathrm{s}]}\end{array}$ & $\begin{array}{l}\mathrm{k} \\
{[]}\end{array}$ & $\begin{array}{l}\omega \\
{[1 / \mathrm{s}]}\end{array}$ & $\begin{array}{l}\lambda \\
{[\mathrm{m}]}\end{array}$ & $\begin{array}{l}\xi \\
{[]}\end{array}$ \\
\hline 1 & 0.0 & 0 & 0.06 & 60.0 & 1.72 & 0.06 & 0.105 & 102.9 & 5.41 \\
\hline 2 & 0.1 & 1 & 0.06 & 60.0 & 1.72 & 0.06 & 0.105 & 102.9 & 5.41 \\
\hline 3 & 0.2 & 2 & 0.06 & 60.0 & 1.72 & 0.06 & 0.105 & 102.9 & 5.41 \\
\hline 4 & 0.3 & 3 & 0.06 & 60.0 & 1.72 & 0.06 & 0.105 & 102.9 & 5.41 \\
\hline 5 & 0.0 & 0 & 0.08 & 60.0 & 1.72 & 0.06 & 0.105 & 102.9 & 4.69 \\
\hline 6 & 0.1 & 1 & 0.08 & 60.0 & 1.72 & 0.06 & 0.105 & 102.9 & 4.69 \\
\hline 7 & 0.2 & 2 & 0.08 & 60.0 & 1.72 & 0.06 & 0.105 & 102.9 & 4.69 \\
\hline 8 & 0.3 & 3 & 0.08 & 60.0 & 1.72 & 0.06 & 0.105 & 102.9 & 4.69 \\
\hline
\end{tabular}

structure develops. Fig. $2 \mathrm{a}$ to $2 \mathrm{~h}$ show the approaching wave and its subsequent interaction with a single obstacle on the beach in top-view images as recorded from the head-mounted CCD camera. Zero time in this paper is referred to the point in time when the wave front reaches the seaward-facing house front. Further, time is normalized by the wave period according to Tab. 1. Once the wave front approaches a single obstacle firstly a shock wave is generated that slowly radiates in the offshore direction while the lee-ward side of the obstacle remains shadowed from the incoming water (Fig. 2a). Between $\hat{t}=0.1-0.2$, the protected lee-ward area is subsequently filled with water and a turbulent wake starts to develop (Figs. 2b, 2c). Meanwhile the first shock wave radiates further offshore and a second shock wave is generated at the front face of the obstacle. Inspection by eye proposes that the second shock wave is yet most probably not caused by the approaching long wave but caused by some minor short waves riding on top of the applied long wave. Those parasitic short waves are product of controller-specific imperfections which is subject to future improvement. Flow velocities at the front of the obstacle generally decreases until approaches zero velocity directly at the obstacle surface. On both sides of the obstacle the shock 


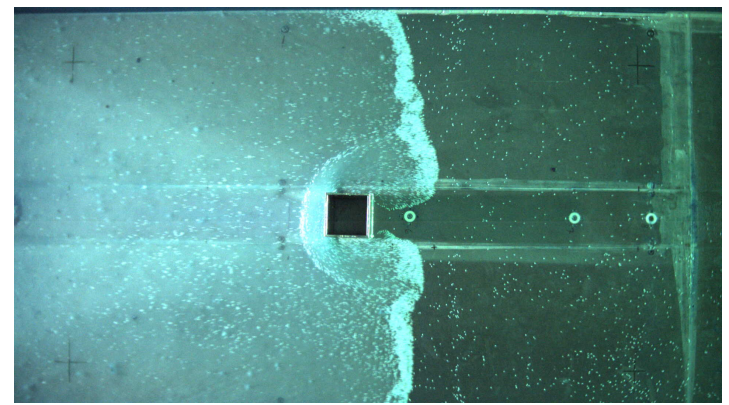

(a) $\hat{t}=0.01$

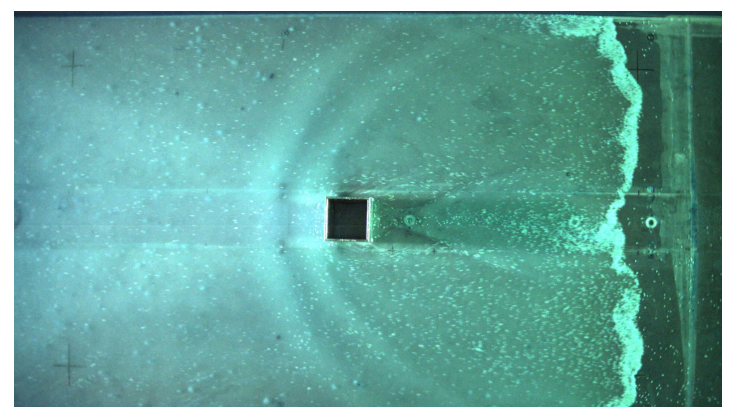

(c) $\hat{t}=0.03$

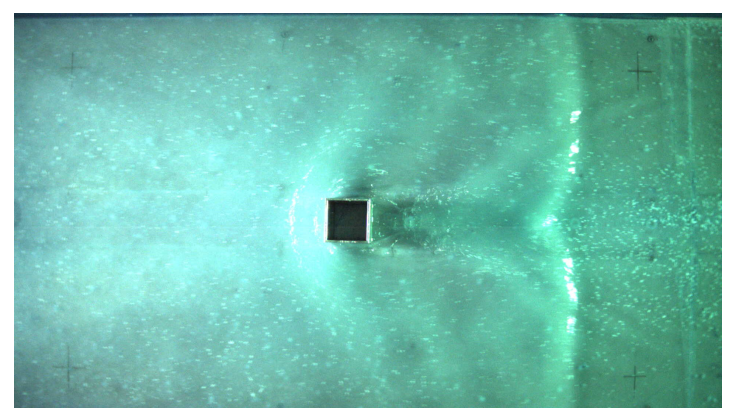

(e) $\hat{t}=0.05$

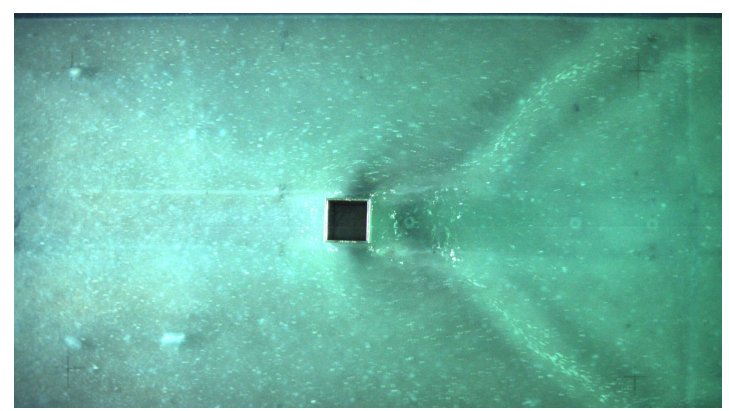

(g) $\hat{t}=0.07$

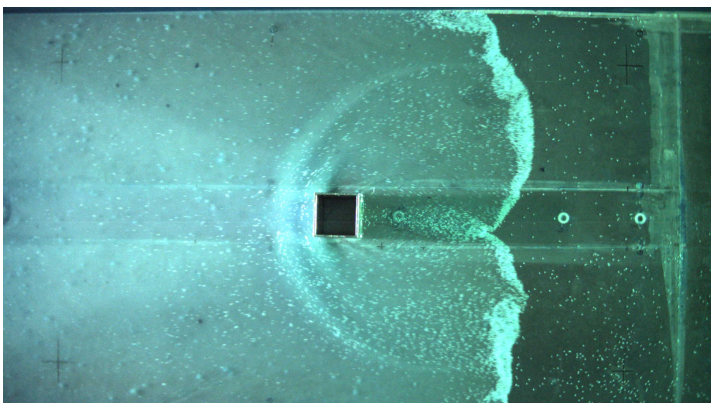

(b) $\hat{t}=0.02$

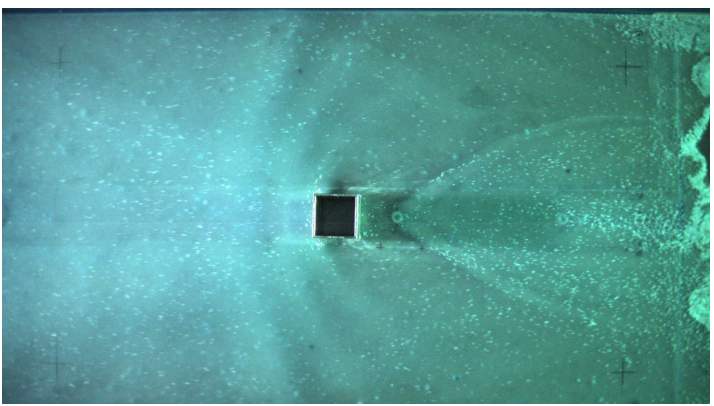

(d) $\hat{t}=0.04$

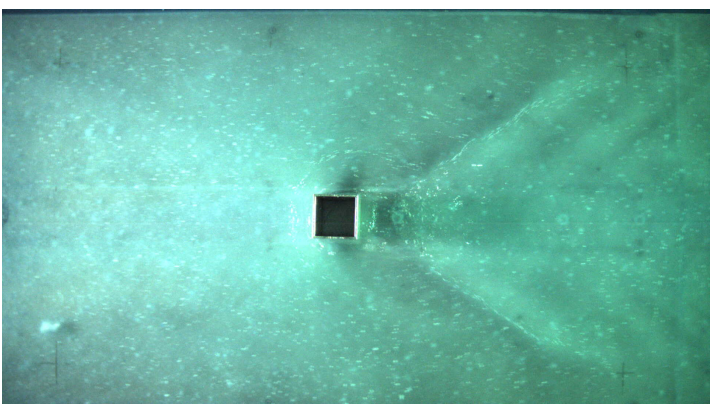

(f) $\hat{t}=0.06$

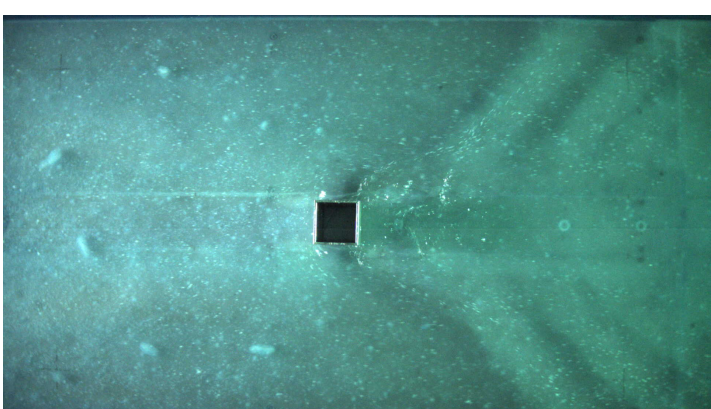

(h) $\hat{t}=0.08$

Figure 2: Sequence of top-view images depicting the fluid-structure interaction of one obstacle during the long wave run-up (direction from left to right) for panels (a) - (h) for normalized time $\hat{t}=\left(t-t_{0}\right) / T$ for gap spacing of $g^{*}=0.0$

wave is advected due to the run-up velocity of the long wave. Figs. $2 \mathrm{~b}$ to $2 \mathrm{~g}$ also highlight how the angle of the wake behind the single obstacle changes over time. The wake angle is defined as the angle between the two visible wake lines that form a V-shape behind the obstacle being exposed the the approach flow. Generally this angle increases over time. More specifically, the wake lines form instationary bows (esp. 
Fig. 2d) as the region of recirculation or abrupt surface elevation change is placed in either a sub- or super-critical flow. Once the wave run-up velocity further decreases, the wake is composed of more than one pair of wake lines.

The above outlined flow features that are present during the wave-structure interaction of a long wave climbing up a plain beach are visually schematized in Fig. 3. In addition to the more apparent features discussed in the previous paragraph, it becomes clear that the front and back edges of the obstacle serve as the main flow separation points. In front of the seaward-facing surface and the inner region behind the obstacle hydraulic jumps form. Turbulence in both regions calms down significantly as the run-up velocity decreases over time.

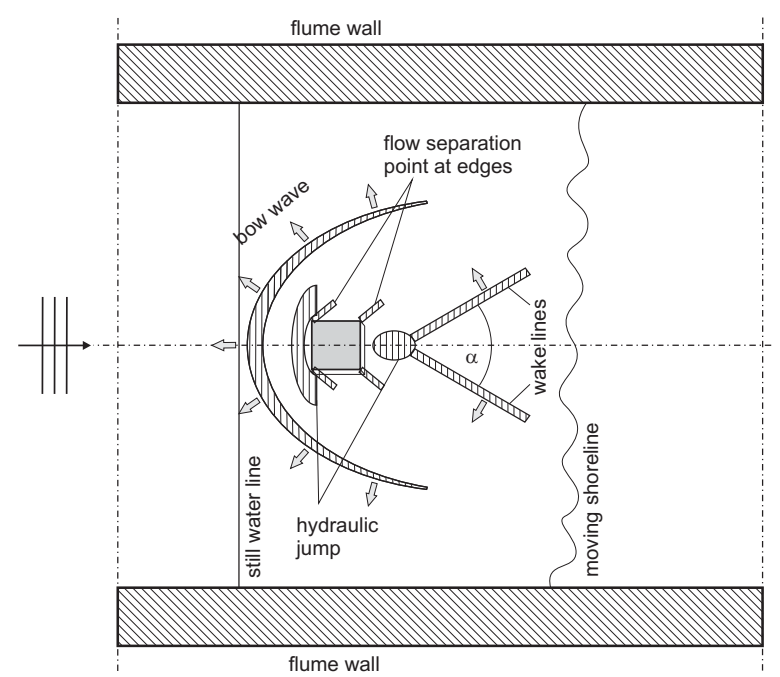

Figure 3: Topological description of the flow features that were observed during the interaction of the long wave with a single free standing vertical structure on a plain beach

Similar to the wave-structure interaction of a single obstacle on a plain beach, distinct flow features evolve when a wave interacts with a pair of obstacles on the beach. A sequence of top-view images is presented in Figs. 4a to 4 h for normalized times of $\hat{t}=0.2,0.4,0.6$ and 0.8 and for two different gap ratios of $g^{*}=1.0$ (left column) and $g^{*}=3.0$ (right column). While general flow features are very similar to those described for the single obstacle interaction, it is apparent, that the pairwise arrangement of the free standing obstacles has its own unique characteristics. Firstly, the evolving bow or shock waves start radiating offshore very similar; however, the generated bow waves merge depending on the gap ratio in the vicinity of the obstacles.

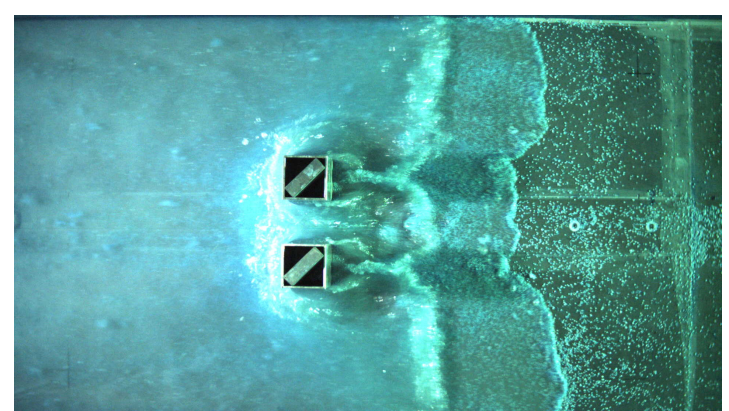

(a) $\hat{t}=0.02, g^{*}=1.0$

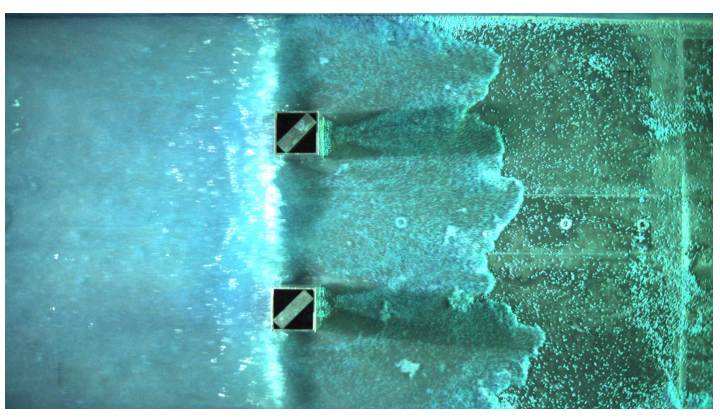

(b) $\hat{t}=0.02, g^{*}=3.0$

Figure 4: Figure continued on next page.

Secondly, a jet-like flow of water is observed in between the gap and beyond in direction of flow which visually appears to change from sub- to supercritical conditions along its path (Fig. 4c, 4e). For 


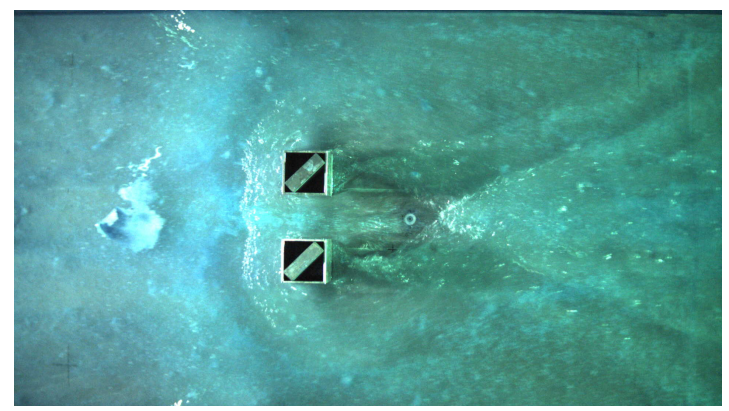

(c) $\hat{t}=0.04, g^{*}=1.0$

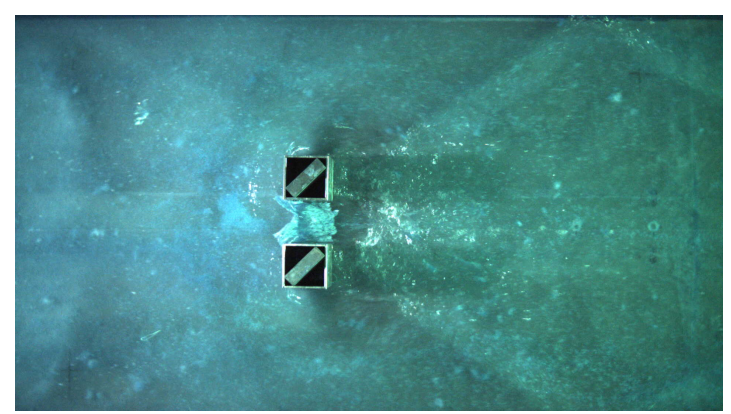

(e) $\hat{t}=0.06, g^{*}=1.0$

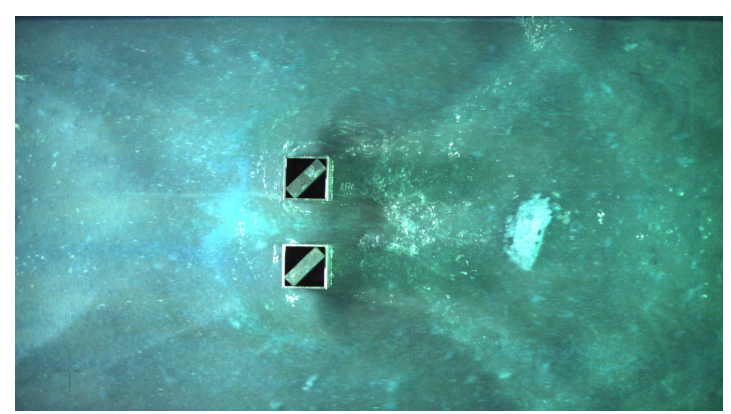

(g) $\hat{t}=0.08, g^{*}=1.0$

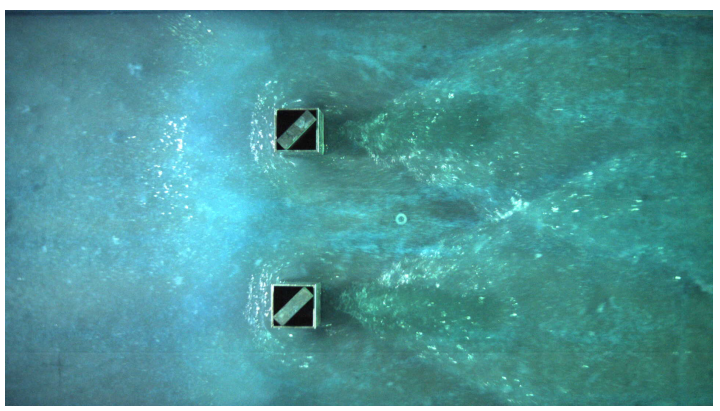

(d) $\hat{t}=0.04, g^{*}=3.0$

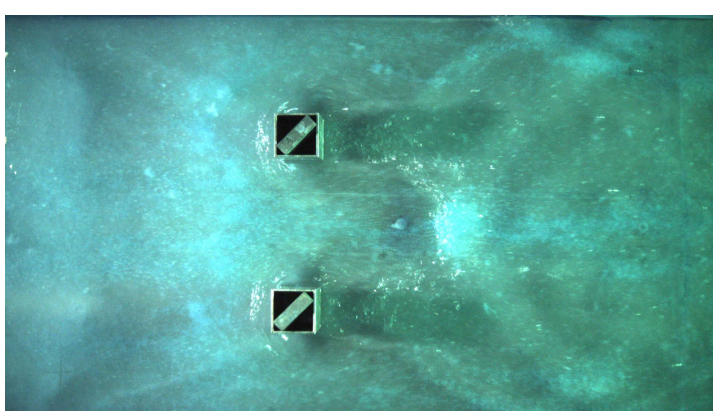

(f) $\hat{t}=0.06, g^{*}=3.0$

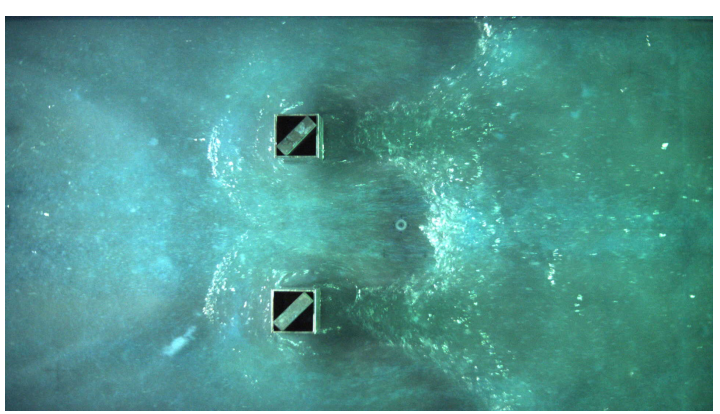

(h) $\hat{t}=0.08, g^{*}=3.0$

Figure 4: Sequence of top-view images depicting the fluid-structure interaction of two obstacles during the long wave run-up (direction from left to right) for panels (a) - (h) for normalized time $\hat{t}=\left(t-t_{0}\right) / T$ for gap ratios of $g^{*}=1.0$ (left column) and $g^{*}=3.0$ (right column)

both gap ratios shown here it becomes clear, that the obstacle arrangement reciprocally influences main flow features. This is namely the inner wake lines being deformed under the influence of the jet generated by the gap formed by the obstacles. This fact is illustrated best in Fig. 4h. Despite this observation, the change of the wake angle over time for two obstacle arrangements has been determined by averaging the respective wake angles for the sake of ease.

\section{RESULTS}

\section{Velocity fields and time series around free standing obstacles}

Velocity fields have been calculated based on a surface PIV algorithm which bases on earlier work from Sveen (2004). Resulting distribution at a relative time step of $\hat{t}=0.013$ is shown in Fig. 5a for a gap ratio $g^{*}=0.0$ and in Fig. $5 b$ for a gap ratio $g^{*}=2.0$. Note that position of virtual wave gauges for below shown time series is indicated by white circle-star symbols. As noted earlier, bow waves with very small to zero velocity are traceable through the velocity fields for both gap ratios. Similarly, shaded lee-side regions where rooster tails and wake lines develop are also visible as low velocity regions. Especially in the turbulent region behind the obstacle, PIV results were very difficult to obtain due to an 
decreased amount of tracers or high degree of turbulence. The bow wave with its low velocity distribution is advected around the obstacles due to the surrounding velocity field in both cases. For the side-by-side arranged obstacles, a jet-like flow develops in between the gap that extends into the back of the obstacles. At the inside front corners of the obstacles flow velocity is almost zero and it increases rapidly following the trajectories of the flow through the gap. This observation is rather unexpected since it is in contrast to continuity considerations. However, the presented flow fields shown in Fig. 5 purely rely on surface PIV analysis and this may help explaining why such a strong velocity gradient at least at the surface exists. It is conjectured that a strong current crosses under the low velocity region near the gap (front) between the obstacles. It is further necessary to continue investigations on 3-D flow conditions during a transient flow motion in the near-field of the infrastructure.

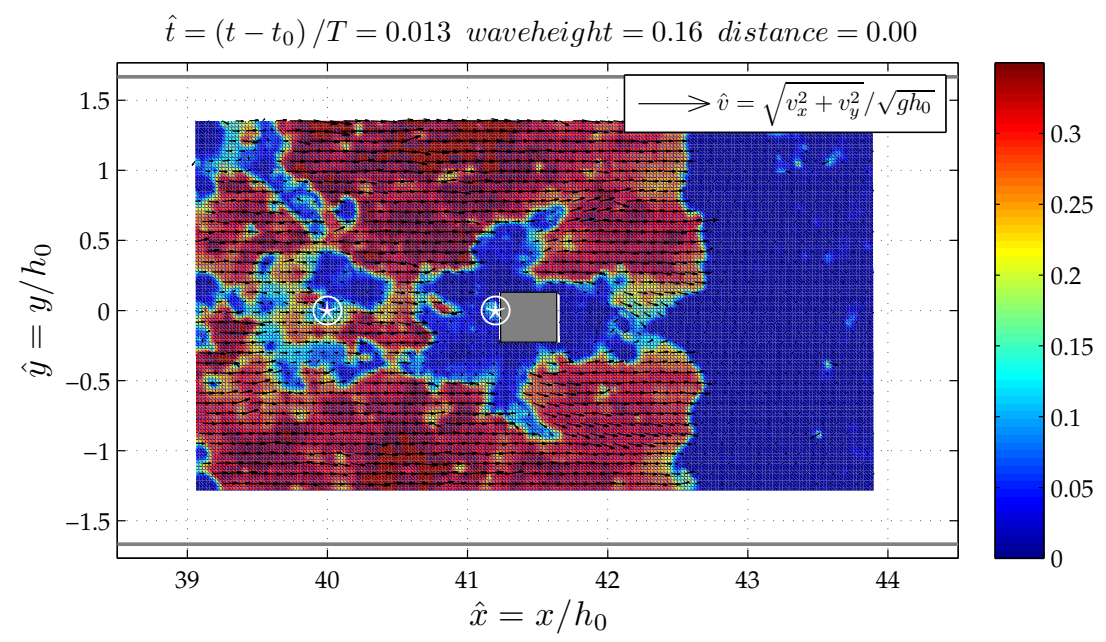

(a) $\hat{t}=0.013, g^{*}=0.0$

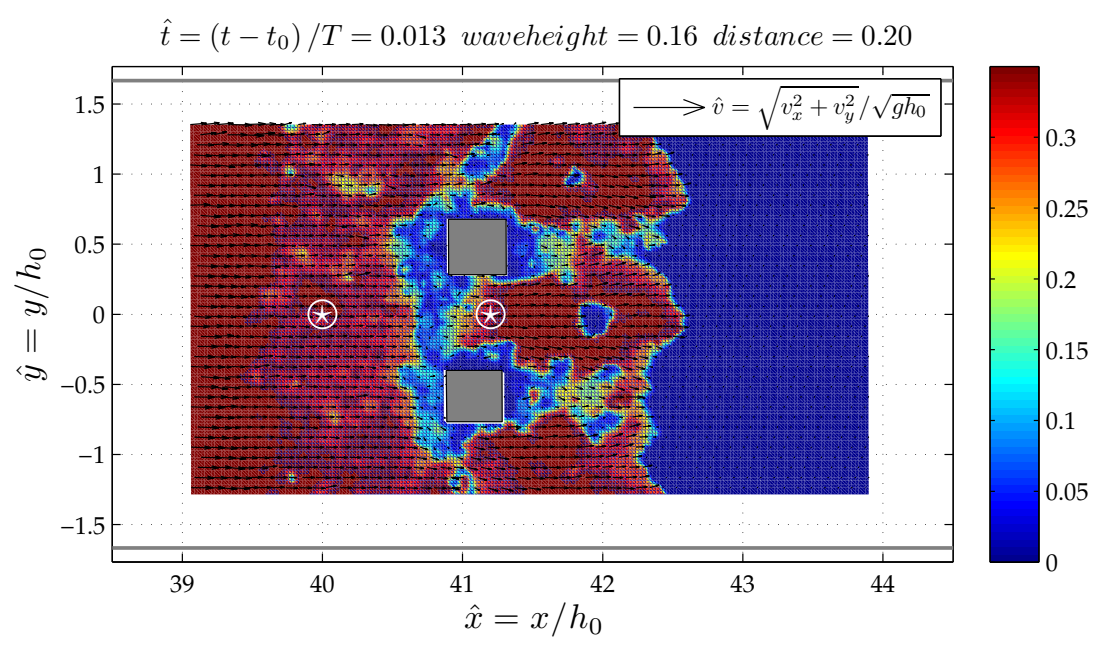

(b) $\hat{t}=0.013, g^{*}=2.0$

Figure 5: Velocity fields derived from surface PIV analysis at a relative time step of $\hat{t}=0.013$ and for two different gap ratios. Virtual gauge positions are indicated by circle-star symbols for later reference.

Fig. 6 presents the development of surface flow velocities for the two presented gap ratios $g^{*}=0.0$ and $g^{*}=2.0$ at the virtual gauge positions indicated in Fig. 5. Velocity time series were assembled from PIV analysis and smoothed with a moving window technique before plotting. Flow velocities decrease with decreasing distance to the obstacle front face significantly, which is coherent with visually obtained description of the flow features presented in Sec. 1. Flow velocity in front of a single obstacle reduces (at least at the surface) up to a stagnation point. Note that velocity magnitudes are not reduced to zero since lateral velocities still happen to drive the uprushing water around the obstacle. 


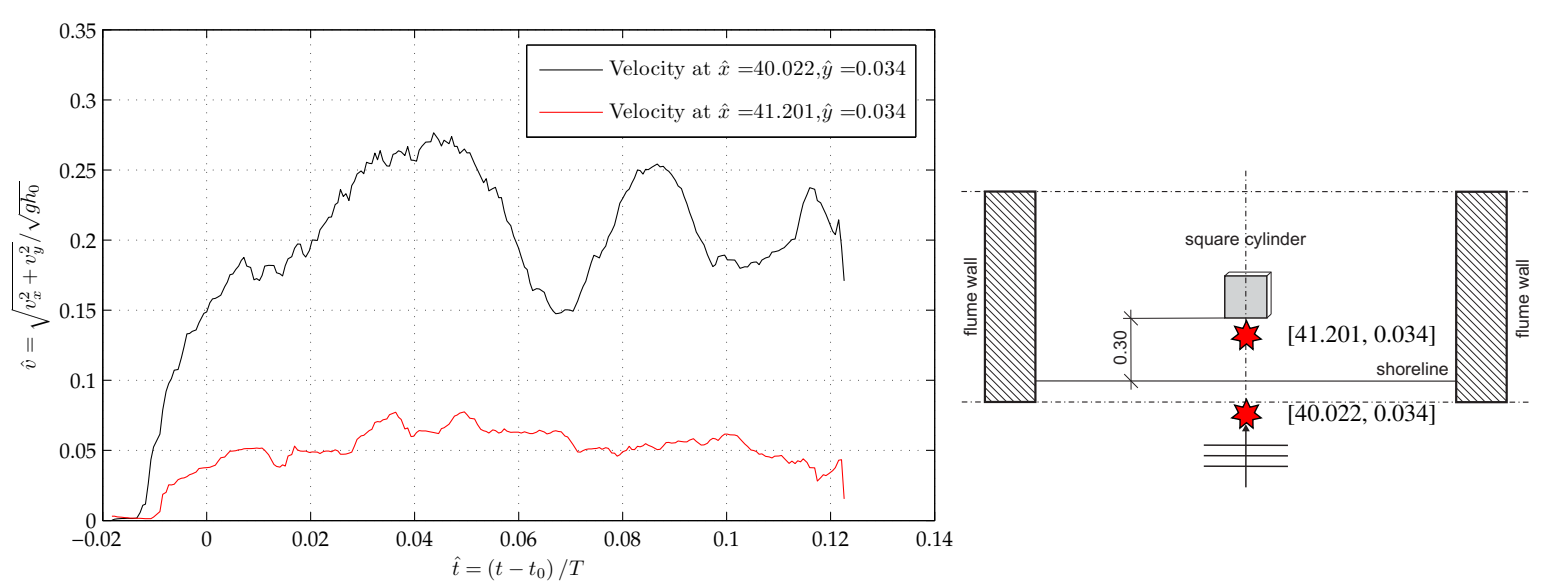

(a) $g^{*}=0.00$ - one obstacle, experiment no. 5 , see Tab. 1

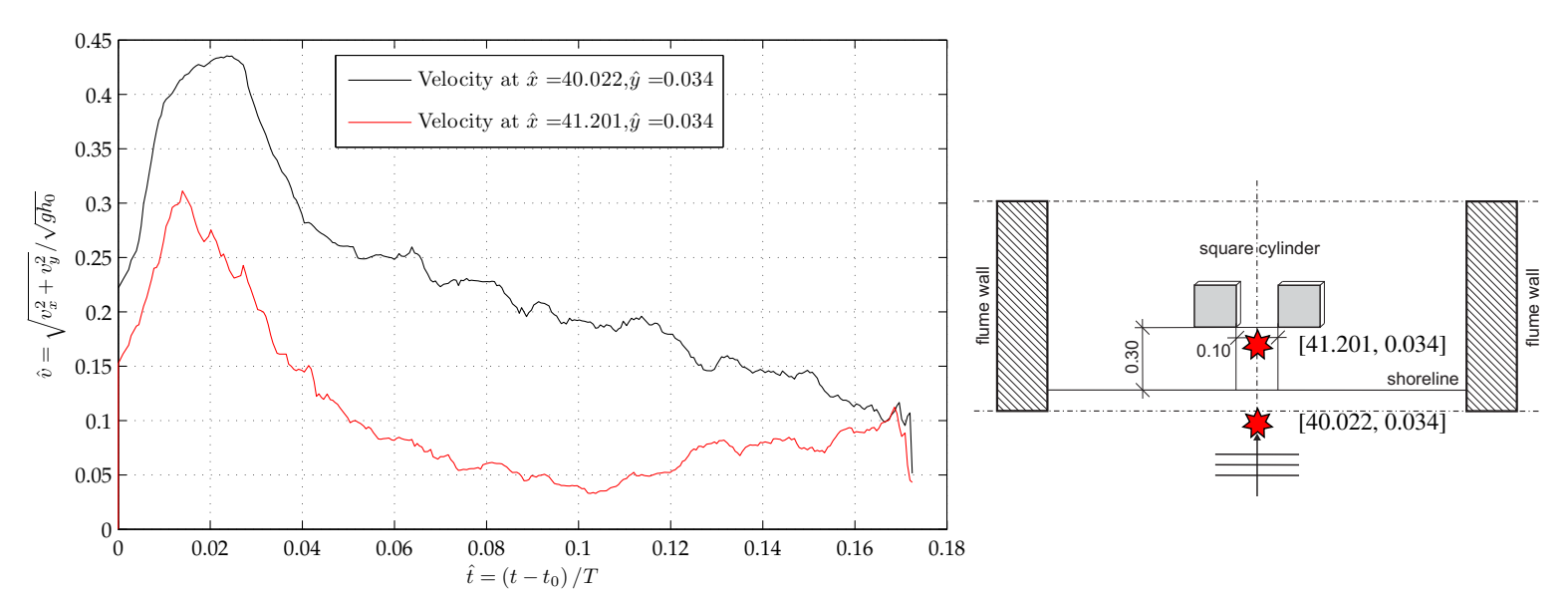

(b) $g^{*}=1.00$ - two obstacles, experiment no. 8 , see Tab. 1

Figure 6: Velocity time series in the vicinity of one obstacle (a) and near side-by-side arranged obstacles (b), all time series with respect to normalized time during wave run-up, for positioning please refer to Sec. 1

Velocity time series at a distance of approximately $0.35 \mathrm{~m}$ (black graph, Fig. 6a) increases very rapidly after the wave front arrives at the structure and slightly oscillates as the parasitic short waves interact with the obstacle; yet the average flow velocity is maintained at $0.2 \mathrm{~m} \mathrm{~s}^{-1}$. Meanwhile, velocity directly at the front face of the obstacle resides at a much lower level around $0.05 \mathrm{~m} \mathrm{~s}^{-1}$ which is a reduction in velocity of almost $25 \%$.

In comparison, velocities along the center axis of the flow problem for the side-by-side arranged obstacles reaches approximately $50 \%$ higher velocities at a distance of $0.35 \mathrm{~m}$, although those velocities last less long and a gradual decrease of average velocities is observable. This general time pattern of velocities along the symmetry axis is also found near the front faces of the obstacles with the difference that peak velocities are decreased by a factor of 50\%. Those near gap velocities are almost as high as those found for the single obstacle case. Although further research is needed to understand the physics of gap flow velocities, it becomes clear that design-wise particular attention has to be paid to side-by-side arranged structures in-situ and guidelines to be adapted.

\section{Rate of change of wake angle}

The evolution of wake lines behind the obstacle(s) may also be of interest for consultants or design engineers in order to prevent neighboring structures to be placed inside the evolving wake region which is characterized by particularly turbulent and potentially dangerous flow. In order to understand the 


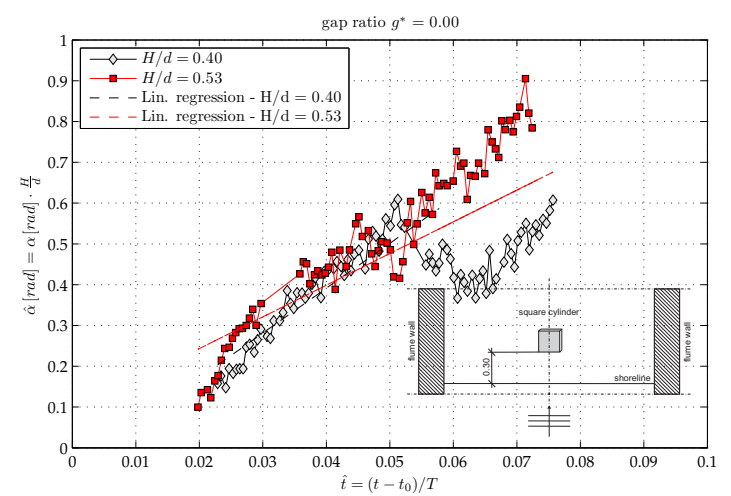

(a) $g^{*}=0.00$ - one obstacle

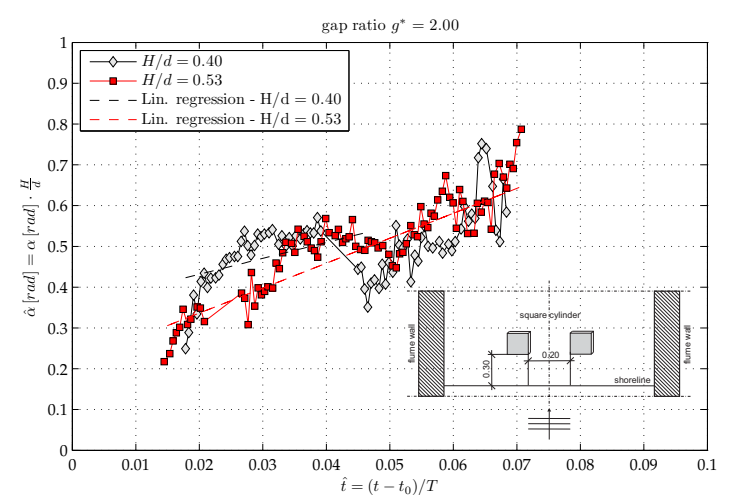

(c) $g^{*}=2.00$ - two obstacles

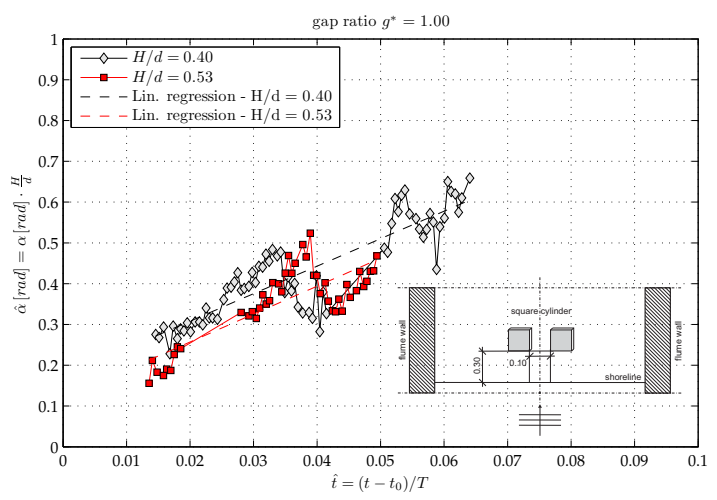

(b) $g^{*}=1.00-$ two obstacles

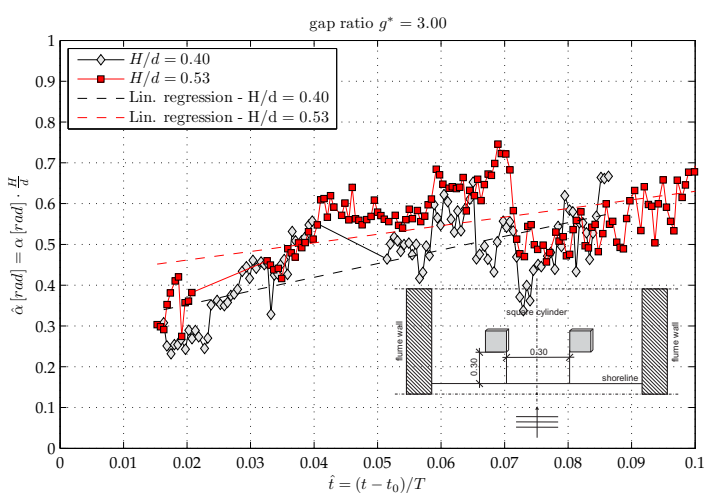

(d) $g^{*}=3.00$ - two obstacles

Figure 7: Temporal evolution of wake angle $\hat{\alpha}$ behind macro-roughness element(s) with respect to normalized time for the tested gap ratios $g^{*}$ during wave run-up

temporal evolution of the wake lines behind the free standing vertical structures while interacting with a long approach wave, wake angles were identified manually from the recorded CCD-camera images. The development of those angles are presented in Figs. $7 \mathrm{a}$ to $7 \mathrm{~d}$.

A continuous increase of the non-dimensional wake angle $\hat{\alpha}$ normalized by the dimensionless wave height $H / d$ occurs which appears to be linear for the tested parameter range. Scatter around the linear regression lines is likely be caused by some parasitic short waves riding on top of the long wave approaching the shore line. A temporary break-down of the evolution of the wake lines could be seen, e.g. in Fig. 7a), gray graph starting at nondimensional time of $\hat{t}=0.05$. Short waves interfering with the main run-up flow are clearly evident from the CCD-camera images. Despite the scatter, good agreement between the experimentally derived wave angles and the regression lines is found. It has to be noted, that the experimental basis does so far not contain wave period variations. As a change in wave period is directly connected to increasing or decreasing run-up velocities it is expected that wake angle also depends on the this wave parameter.

From the previous findings it was possible to derive growth rates of the wake angles with respect to the tested range of gap ratios. As plotted in Fig. 8, a linear reciprocal correlation exists between the rate of growth of the wake angle $\hat{\alpha} / \hat{t}$ and the gap ratio $g^{*}$. As growth rates generally decrease with increasing gap ratio, it cannot be answered on the basis of the results presented herein at which gap ratio the influence of the side-by-side arranged obstacles ceases; thus, the distance at which both obstacles would react individually has not been found yet due to width restrictions of the applied flume. However, Eq. (2) or alternatively Eq. (3) allow for a prediction of the growth rates of the wake angles within the given experimental limits indicated in Fig. 8. 


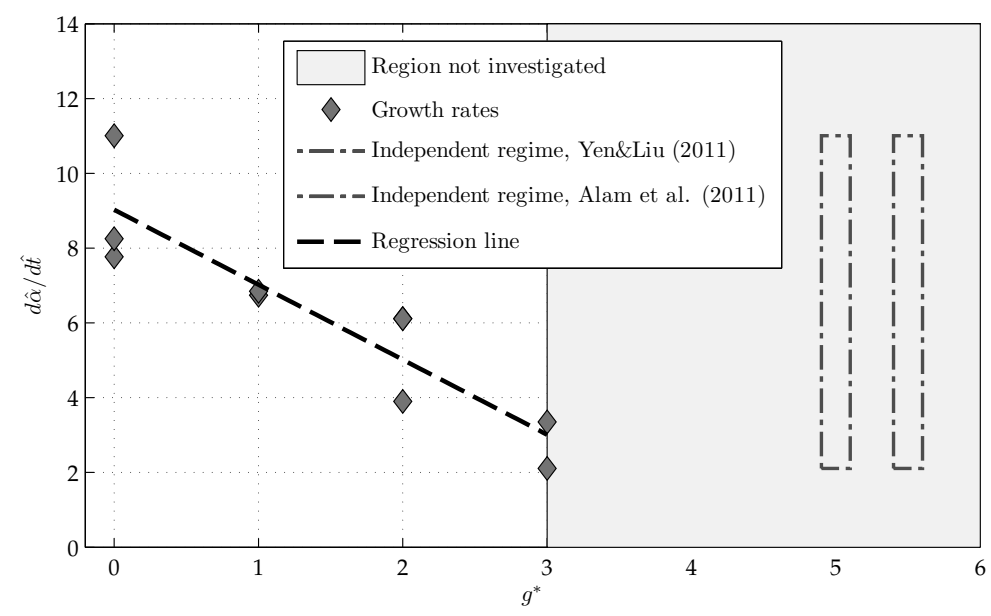

Figure 8: Linear rate of change for wake angle with respect to the tested gap ratios, region beyond $g^{*}>3.0$ was not tested for experimental reasons. Filled diamonds indicate growth rates form individual experiments, dashed line represents the regression line according to Eq. (2) or (3).

Dimensionless growth rate of the wake angle $\hat{\alpha} / \hat{t}$ with respect to the gap ratio is given as follows:

$$
\frac{d \hat{\alpha}}{d \hat{t}}=-2.0 g^{*}+9.03
$$

where $g^{*}$ is the gap ratio.

Alternatively, Eq. (2) could be expressed in dimensional form accordingly:

$$
\frac{d \alpha}{d \hat{t}}=\left(-2.0 g^{*}+9.03\right) \frac{d}{H}
$$

where $d / H$ is the inverse of the relaive wave height.

\section{SUMMARY AND CONCLUSIONS}

The objective of this study was the description of a transient approach flow interacting with a single or side-by-side arranged free vertical structures. From the existing literature only very few authors addressed the stationary nature of approach flows appearing i.e. during the attack of a tsunami wave or a dam break wave around vertical structures. The specific flow characteristics of such a flow has been qualitatively described for either single or side-by-side arranged obstacles. The topological description of the flow features most importantly revealed bow or shock waves radiating offshore from the obstacle(s) while stagnation regions as well as hydraulic jumps occur around the structures.

In addition, the nature of the wake evolution has been described qualitatively and quantitatively. As wake regions behind structures depict potential for destruction of neighboring structures, the evolution has been characterized by the change of wake angle in time and the growth rate of the wake angle has been determined. So far, it is still unanswered how far, the influence of the wake reaches behind the structure under investigation. This is yet also dependent on the slope of the beach and further experimental or numerical research should be initiated. Furthermore, uncertainty exists, at which gap ratio no mutual influence between side-by-side arranged obstacles is exerted. Yen and Liu (2011) reports that two independent vortex streets occur for gap spacings $g^{*}<6.0$ with in-phase vortex shedding. In addition, Alam et al. (2011) denotes that effects on drag coefficients of air flow around square cylinders vanish for values $T / d=(g-w) / d>5.0$ (note that their definition of the gap spacing is lightly different). Unfortunately, width limitations of the applied wave flume prevented measurements at such high gap ratios and further research is also needed to reduce this uncertainty in detail.

\section{ACKNOWLEDGMENTS}

The authors gratefully acknowledge the support of N. Lange who contributed to laboratory work and subsequent analysis in the framework of her master thesis. 


\section{REFERENCES}

Alam, M., Y. Zhou, and X. Wang (2011). "The wake of two side-by-side square cylinders". In: Journal of Fluid Mechanics 669, pp. 432-471.

Ayukawa, K., J. Ochi, G. Kawahara, and T. Hirao (1993). "Effects of shear rate on the flow around a square cylinder in a uniform shear flow". In: Journal of Wind Engineering and Industrial Aerodynamics 50.C, pp. 97-106.

Cox, D., T. Tomita, P. Lynett, and R. Holman (2009). "Tsunami inundation with macro-roughness in the constructed environment". In: Proceedings of the International Conference on Coastal engineering. Ed. by J. M. Smith. Vol. 2. World Scientific, pp. 1421-1432.

Cui, X. and J. Gray (2013). "Gravity-driven granular free-surface flow around a circular cylinder". In: Journal of Fluid Mechanics 720, pp. 314-337.

Dargahi, B. (1989). "The turbulent flow field around a circular cylinder". In: Experiments in Fluids 8.1-2, pp. $1-12$.

Goseberg, N. (2012). "A laboratory perspective of long wave generation". In: Proceedings of the International Offshore and Polar Engineering Conference, pp. 54-60.

- (2013). "Reduction of maximum tsunami run-up due to the interaction with beachfront development - application of single sinusoidal waves". In: Natural Hazards and Earth System Science 13.11, pp. 2991-3010.

Goseberg, N. and T. Schlurmann (2012). "Interaction of idealized urban infrastructure and long waves during run-up and on-land flow process in coastal regions". In: Proceedings of the International Conference on Coastal Engineering. Ed. by P. Lynett and J. M. Smith. Santander, Spain.

Goseberg, N., A. Wurpts, and T. Schlurmann (2013). "Laboratory-scale generation of tsunami and long waves". In: Coastal Engineering 79, pp. 57-74.

Li, R., S. Liu, Q. Guan, and Y. Peng (2011). "Post-disaster assessment of northeastern coastal region for the 2011 Sendai Earthquake and tsunami”. In: ISWREP 2011 - Proceedings of 2011 International Symposium on Water Resource and Environmental Protection 3, pp. 2429-2432.

Malavasi, S. and A. Guadagnini (2007). "Interactions between a rectangular cylinder and a free-surface flow". In: Journal of Fluids and Structures 23.8, pp. 1137-1148.

Mignot, E. and N. Riviere (2010). "Bow-wave-like hydraulic jump and horseshoe vortex around an obstacle in a supercritical open channel flow". In: Physics of Fluids 22.11.

Pagliara, S., S. Kurdistani, and T. Roshni (2011). "Rooster tail wave hydraulics of chutes". In: Journal of Hydraulic Engineering 137.9, pp. 1085-1088.

Singha, A. and R. Balachandar (2011). "Structure of wake of a sharp-edged bluff body in a shallow channel flow". In: Journal of Fluids and Structures 27.2, pp. 233-249.

Sousa, J. (2002). "Turbulent flow around a surface-mounted obstacle using 2D-3C DPIV". In: Experiments in Fluids 33.6, pp. 854-862.

Sumer, B., N. Christiansen, and J. Fredsoe (1997). "The horseshoe vortex and vortex shedding around a vertical wall-mounted cylinder exposed to waves”. In: Journal of Fluid Mechanics 332, pp. 41-70.

Sveen, J. K. (2004). An introduction to MatPIV v. 1.6. 1. Preprint series. Mechanics and Applied Mathematics.

Taubenböck, H., N. Goseberg, G. Lämmel, N. Setiadi, T. Schlurmann, K. Nagel, F. Siegert, J. Birkmann, K.-P. Traub, S. Dech, V. Keuck, F. Lehmann, G. Strunz, and H. Klüpfel (2013). "Risk reduction at the "Last-Mile": An attempt to turn science into action by the example of Padang, Indonesia". In: Natural Hazards 65.1, pp. 915-945.

Taubenböck, H., N. Goseberg, N. Setiadi, G. Lämmel, F. Moder, M. Oczipka, H. Klüpfel, R. Wahl, T. Schlurmann, G. Strunz, J. Birkmann, K. Nagel, F. Siegert, F. Lehmann, S. Dech, A. Gress, and R. Klein (2009). "Last-Mile preparation to a potential disaster - Interdisciplinary approach towards tsunami early warning and an evacuation information system for the coastal city of Padang, Indonesia". In: Nat Hazard Earth Sys 9.4, pp. 1509-1528.

Wong, P., N. Ko, and A. Chiu (1995). "Flow characteristics around two parallel adjacent square cylinders of different sizes". In: Journal of Wind Engineering and Industrial Aerodynamics 54-55.C, pp. 263-275.

Yen, S. and J. Liu (2011). "Wake flow behind two side-by-side square cylinders". In: International Journal of Heat and Fluid Flow 32.1, pp. 41-51. 